\title{
Unraveling the soul of autoimmune diseases: pathogenesis, diagnosis and treatment adding dowels to the puzzle
}

\author{
S. Colafrancesco $\cdot$ N. Agmon-Levin • \\ C. Perricone $\cdot$ Y. Shoenfeld
}

Published online: 4 June 2013

(C) Springer Science+Business Media New York 2013

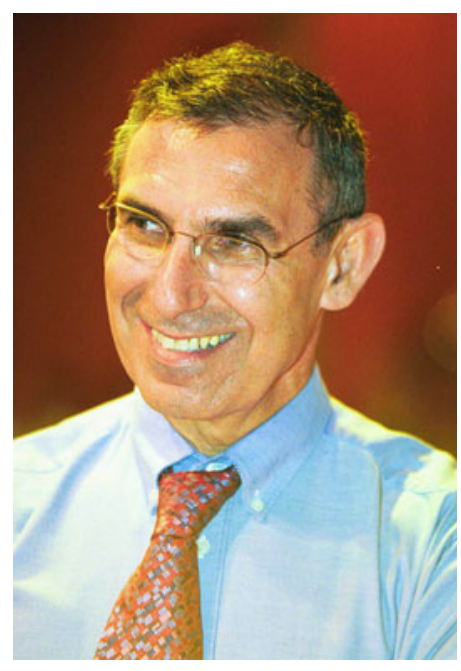

Y. Shoenfeld

\begin{abstract}
The pathogenesis of autoimmune diseases (ADs) is characterized by a complex interaction between genetic, immune defects, environmental and hormonal factors. The concept of "mosaic of autoimmunity" deals with the multifactorial origin and diversity of expression of ADs in humans. Genetic leads to a predisposition in developing an autoimmune syndrome, but the presence of an external or endogenous environmental factor, recently called "exposome," is essential in triggering the immune response. Infections as well as the expositions to different other external environmental agents have been identified as potential trigger for ADs. A new syndrome, namely the autoimmune/inflammatory syndrome induced by adjuvants, has recently been defined alluding to the key role of adjuvant in inducing an immune-mediated condition. Aluminum and silicone, respectively found in vaccines and breast implants, are the most commonly known adjuvants charged with development of autoimmune conditions. Similarly to playing chess, unraveling the pathogenesis of autoimmune diseases with every new discovery is adding a move to the game aiming at checkmating ADs.
\end{abstract}

Keywords Autoimmunity · Autoantibodies · Autoimmune/inflammatory syndrome induced by adjuvants (ASIA) . Genetic $\cdot$ Infections $\cdot$ Aluminum $\cdot$ Silicone

Autoimmune diseases (ADs) are chronic conditions initiated by the loss of immunological tolerance to self-antigens. In the pathogenesis of ADs, a complex interaction between genetic, immune defects, environmental and

S. Colafrancesco · N. Agmon-Levin · C. Perricone .

Y. Shoenfeld $(\bowtie)$

The Zabludowicz Center for Autoimmune Diseases, Sheba

Medical Center, 52621 Tel Hashomer, Israel

e-mail: Shoenfel@post.tau.ac.il

S. Colafrancesco - C. Perricone

Reumatologia, Dipartimento di Medicina Interna e Specialità

Mediche, Sapienza Università di Roma, Rome, Italy

\section{N. Agmon-Levin}

Sackler Faculty of Medicine, Tel Aviv University, Tel Aviv, Israel

Y. Shoenfeld

Incumbent of the Laura Schwarz-Kip Chair for Research of

Autoimmune Diseases, Tel-Aviv University, Tel Aviv, Israel hormonal factors has been hypothesized [1]. The so-called mosaic of autoimmunity [2] encompasses a multi-factorial origin and diversity of expression of ADs in humans. The mechanisms underlying such conditions are not still clarified. This special issue of Immunological research is focused on the rising aspects on pathogenesis, diagnosis and treatment of autoimmune diseases. Selected papers from the 8th International congress on Autoimmunity that was held in Granada in May 2012 have outlined the importance of unravelling the different facades of autoimmunity. Genetic leads to a predisposition in developing an autoimmune or an autoinflammatory syndrome, but the presence of an external or endogenous environmental factor, recently called "exposome" [3], is essential in triggering the immune response. Perricone et al. [4] have stressed that a specific genetic background seems to be required for the development of an autoimmune response. Indeed, already a number of gene polymorphisms have been associated with the onset of ADs [4]. Nonetheless, 
since the production of autoantibodies is genetically determined and their presence seems to determine the clinical presentation of ADs, the authors raised the question whether genetic predisposes to the development of the autoantibody rather than to overt disease. For instance, concerning the genetic predisposition to rheumatoid arthritis (RA), there are a number of evidences dealing with the association between HLA shared epitope, smoke, the production of anti-citrullinated protein/peptide antibodies and the disease susceptibility and severity [5]. Genes may favor also the onset of disease comorbidities, such as cardiovascular (CV) involvement. A recent study has underlined the connection between RA and an increased risk of CVD, which is driven by both traditional risk factors (hypercholesterolemia, diabetes mellitus, abnormal body mass index, abdominal obesity and smoking) and non-traditional risk factors (familial autoimmunity, more than 10 years of duration of the disease, age at onset, patients who worked at household duties, use of systemic steroids and low educational level) [6]. Thus, it is clear that environmental factors are driving the autoimmune process, of which smoke habits is one of the most known risk factor [6]. Cigarette smoke leads to a release of intracellular antigens via tissue hypoxia or toxin-mediated cellular necrosis, and its products can enhance autoreactive B cell or peripheral T-lymphocytes proliferation. Bieber et al. [7] have reported that the subsequent production of free radicals interacting with DNA may cause genetic mutations and elicit a gene activation that leads to ADs, suggesting the overlap between RA and chronic obstructive pulmonary disease. Parallel to smoke, infections are known to play a major role. For this reason, Bogdanos et al. [8] have included in the field of "exposome" the new concept of the "infectome" meant as the study of all infectious factors which contribute to the development of an autoimmune disease. The pathogenic association between infectious agents and the development of ADs is well known and carefully reviewed in the literature [8,9]. Specifically, the infectious agent can trigger an autoimmune response through different mechanisms including molecular mimicry, epitope spreading, bystander activation and polyclonal activation $[10,11]$. On the other hand, investigations of the immunomodulatory effects operated by parasitic infections have documented a protective effect of some parasitic infections against autoimmune inflammation. This beneficial effect is believed to involve the shift toward a Th2 predominant immunological phenotype [12]. In this view, Fisher et al. [13] reported a significantly higher seroprevalence of anti-Toxoplasma gondii $\mathrm{IgG}$ antibody in European patients with RA compared to European patients with SLE, suggesting a plausible role of Toxoplasma gondii infection in the pathogenesis of RA. This possibility opens a new chapter dealing with prevention or treatment of patients with RA by eradicating $T$. gondii infection. In the context of parasite infections, the role of eosinophils in ADs is still unclear. Indirect data derive from an emerging disease eosinophilic esophagitis (EoE), in which a major pathogenic role is played by an altered immune response, non-IgE mediated, that winks to autoimmunity [14]. In another study, Dolcino et al. [15] found that anti-rotavirus antibodies may predict the onset of celiac disease suggesting a pathogenic role for this virus [15]. It is clear that again the genetic background is necessary (but not sufficient) for the development of overt disease, probably involving mechanism of protection from infection as recently demonstrated in Ashkenazi individuals [16].

A new star within the external environmental agents in triggering ADs is the adjuvant. An entirely new syndrome, namely the autoimmune/inflammatory syndrome induced by adjuvants (ASIA) [17-20], has been defined. This syndrome includes several medical conditions (vaccination-induced autoimmunity [21], silicone implants-induced autoimmunity [22], Gulf War Syndrome (GWS) [23], macrophagic myofasciitis (MMF) with chronic fatigue syndrome [24] and the sick building syndrome [25]) which share as common denominator the presence of an adjuvant that chronically stimulates the immune system [26]. Adjuvants have been used for decades to improve the immune response to vaccines, and among this large group, aluminum and silicone are the most commonly described. Nonetheless, as supported by increasing reports [27, 28], although rarely vaccines are able to trigger the development of ADs in genetically susceptible humans, this could be ascribed to the presence of containing adjuvants [29-31]. The time relationship between the vaccine delivery and overt disease can last from few weeks to even years. Interestingly, Cerpa-Cruz et al. [32] confirmed the link between vaccinations and increased risk of autoimmune responses suggestive for ASIA. In this study, 43 out of 120 patients experienced the new onset of a nonspecific inflammatory response (fever, myalgia, arthralgia) or signs or symptoms of an immune-mediated condition following vaccination.

In the last century, aluminum became available for human use not only as vaccine adjuvant but also as a common additive in various processed foods, cosmetics or pharmaceutical products. A now abundant literature shows that exposure of humans and animals to aluminum from various sources can have deleterious consequences on nervous system, especially in adults [33]. This fact was extensively addressed by Shaw and Tomljenovic [33] who described the association between aluminum exposure and amyotrophic lateral sclerosis, Alzheimer's disease or autism spectrum disorders [34]. In Alzheimer's disease, in addition to amyloid $\beta$ accumulation, high levels of aluminum in the brain of patients have been found. Moreover, a correlation between the number of vaccines delivery 
containing aluminum and the changes in autism rates as well as the detection of higher than normal aluminum levels in the hair, blood and/or urine of autistic children has been described [35]. We should underline that the mechanism by which aluminum is able to induce neurological disorders is probably dual. Indeed, aluminum can both directly damage the blood-brain barrier thus increasing its permeability [36], or indirectly stimulate the release of proinflammatory cytokines from microglia [37], or up-regulate chemo-attractants responsible for the recruitment of immunocompetent cells to the brain [38]. Luján et al. [39] went further and reported the potential of aluminum to trigger neurological disorders in an animal model [39]. In this study, a newly recognized entity similar to the ASIA syndrome in humans following immunization has been described in sheep. This study points the evidence that neurological immune-mediated damage caused by aluminum can be observed in in vivo models of disease.

Indeed, Gherardi et al. [40] described a severe meningoencephalitis probably due to the direct transport of aluminum by macrophages to the central nervous system. In a recent paper, authors from the same group were able to identify a CCL2-dependent pathway of penetration of alum into the brain [40]. Such transfer of aluminum to draining lymph nodes, spleen and brain operated by monocyte-lineage cells has been described in patients with MMF [41]. It is interesting to know that an analogue evidence of translocation operated by macrophages has been found also for silicon. Since this substance was considered an inert material and thus unable to induce immune reactions [42], silicon was incorporated into a myriad of medical products. Such molecule is most commonly used as component of breast implants and once injected it is able to stimulate a local macrophage activation which results in cytokines and reactive oxygen species production. The process of oxidation leads to macrophage apoptosis resulting in the release of silicon containing particles that can be taken up once again by other macrophages that amplify the inflammatory process [43]. This condition carries to the silica formation or, alternatively, to the silicon translocation to the regional lymph nodes [44]. The property of silicon to trigger the immune response and induce autoimmunity has been already described [45, 46]. Cohen Tervaert et al. [47] reported on a series of 32 patients with silicone breast implants who developed immune-mediated conditions. If half of the patients were diagnosed with a well-defined systemic autoimmune disease, all patients suffered from a constellation of sign and symptoms and fulfilled the proposed criteria for ASIA.

Diagnosing autoimmunity has been a medical challenge for decades. One of the mainstay of such diagnostic process is autoantibodies testing. Indeed, the search and quantification of autoantibodies are critical in classifying several autoimmune diseases and are helpful in performing differential diagnosis. In this special issue one particular eye has been given not only to the different diagnostic techniques but also to the potential biomarkers for autoimmune diseases. Despite autoimmune diseases have conventionally been considered as distinct disorders and, consequently, separately treated, the concept of autoimmune comorbidity has been introduced. Indeed, multiple autoimmune syndromes and overlap syndromes can coexist in the same patient. The importance of multiple autoantibodies testing to diagnose such comorbidities has been outlined by Tozzoli et al. [48]. The authors stressed that autoimmune patients at higher risk for the development of polyautoimmunity (i.e. a second autoimmune disease) are candidates for extended serological testing as well as careful clinical evaluation. The cost-effective ratio of such behavior should be evaluated. In this view, it would be important to: (1) unravel the most commonly overlapping antibodies and most specific; (2) support the development of automated and standardized multiplex immunoassays carefully selecting specific kits for most likely overlapping syndromes. The assays should always careful give the quantification of the searched autoantibody [49]. Indeed, this is a clinically useful practice, not only in the diagnostic stage, but also during follow-up of patients with autoimmune diseases. It is general opinion that the highest is the titer of the antibody, more likely the patient will be affected with the respective autoimmune disease. However, there are several cases in which the level of the antibody is not crucial in predicting disease relapses. This is the case of anti-citrullinated protein/peptide antibodies (anti-CCP) that in several studies are reported unmodified after anti-TNF therapy in patients with RA despite disease activity amelioration $[50,51]$. It is possible that the test used to assess anti-CCP can influence these results. For instance, Szekanecz et al. [52] have found that the CCP3.1 test shows better performances when compared with $\mathrm{CCP} 2$ and MCV in patients with seronegative RA. This evidence may in some cases even change the classification of a patient (according with the new criteria), and it cannot be excluded that when applied to monitoring of disease status and response to therapy, this test may show superior sensitivity. Thus, it is clear that the laboratory autoimmunologist should be put in the position to have precise instruments supporting the clinical data to better address the disease status of the patients. In this view, the assessment of the bishops within the autoantibodies, the anti-nuclear antibodies (ANA) is crucial. Indeed, till nowadays standardization of ANA measurement is lacking and automated methods are not fully reliable. Thus, Zafrir et al. [53] evaluated an automated chemiluminescent immunoassay kit for ANA, the LIAISON ${ }^{\circledR}$ (DiaSorin, Italy). They found that the specificity for ANA screening was $97 \%$ in a cohort of patients with different ADs, suggesting that the 
kit is specific and sensitive for the evaluation of ANA in patients with primary biliary cirrhosis, scleroderma and Sjögren's syndrome. The possibility to finally have standardized measures is just out the door and waiting to be confirmed in larger populations.

Moreover, there are cases in which the role of antibodies has been for too long underestimated. As such the antiphosphatidylserine/prothrombin antibodies have not been lucky in the field of autoimmunity, despite the fact that they represent two-thirds of lupus anticoagulant (LA) prothrombotic activity and that they can be quite easily detected by ELISA. In this special issue, Pregnolato et al. [54] found a high prevalence of anti-phosphatidylserine/ prothrombin antibodies in LA-positive subjects (81-87 \%) and, more relevantly, a strong correlation with thrombotic manifestations. If further validated, it could be suggested to include these antibodies within the major criteria for classification of antiphospholipid syndrome (APS) and to evaluated the cost-effectiveness of such measure.

Besides autoantibodies, a major pathogenic and diagnostic role in autoimmunity is still played by complement. Ballanti et al. [55] have extensively reviewed the functions and implications of this complex system. We should remind that complement is included in the assessment of disease activity in systemic lupus erythematosus (SLE). Ballanti et al. [50] have addressed on recent data suggesting that complement levels may be crucial also in other conditions, such as psoriatic and rheumatoid arthritis in the monitoring of anti-TNF therapy [56].

Rising expectation are awaited from the study of the "abzymes," those antibodies executing catalytic activity, in particular those with deoxyribonuclease (DNase) activity. Their pathogenic and diagnostic role in ADs is still unclear, but a new chapter in autoimmunity has been opened [57]. A number of molecules are instead gaining interest for their potential as disease biomarkers. For instance, pentraxin 3 (PTX3) has been recognized as a marker of early joint inflammation in patients with juvenile idiopathic arthritis (JIA). Padeh et al. [58] characterized this acute phase protein in synovial fluid (SF) of patients with JIA and found that PTX3 levels were related to arthritis exacerbation and in vitro stimulation of cells from a synovial cell line. It is very interesting to learn that PTX3 may play a major role in early stages of inflammation, possibly suggesting a potential role for this molecule in disease prediction thus prevention. PTX3 is a cardiovascular biomarker, once more stressing the close relationship between mechanisms underlying arthritides and the autoimmune/inflammatory condition atherosclerosis [59].

Coming to an end, the recent acquisitions of science lead to great progresses also in the treatment of ADs. First, we are observing the usage of old drugs in new diseases or for other purposes than initially meant. For instance, Khattri and
Zandman-Goddard [60] have revised the role of statins in ADs underlying not only the beneficial effects on $\mathrm{CV}$ involvement, but also in ameliorating disease activity due to their immunoregulatory properties. Katzav et al. [61] have suggested the potential to treat patients with experimental autoimmune neuritis with clodronate. The longer steps have been jumped with the upcoming of biologics. Indeed, monoclonal antibodies anti-interleukin (IL) 6 seems promising in the treatment of Sjögren's syndrome [62], refractory pemphigus foliaceus and Behçet's disease [63]. At the same extent, rituximab, successfully used RA and in some lymphomas, has been proven beneficial in refractory idiopathic inflammatory myopathies and antisynthetase syndrome [64]. In the usage of these drug, careful attention should be given to the other side of the coin, represented by the possible occurrence of autoimmune complications with rise of autoantibodies and pro-inflammatory molecules as Lora et al. [65] have reported. In the future, the best bet could be on staminal cells and mesenchymal stromal cells [66]. In the intriguing and mysterious field of chronic fatigue syndrome/ myalgic encephalomyelitis, a new hope is represented by immunostimulation [67] aimed at eradicating the pathogenic agents triggering the abnormal immune response observed in these conditions. Since we have abovementioned the importance of autoantibodies in the development of ADs, it is known the importance of their clearance as a possible therapeutic strategy. This was recently addressed by Hershko et al. [68] who analyzed a new tool, Lupusorb, as an effective modality for eliminating anti-VRT-101 antibodies. We have also to learn how to treat and for how long. This is the case of APS, in which discontinuation of anticoagulation or antiaggregation treatment is still a matter of debate. Coloma Bazar et al. [69] showed that treatment can be stopped in patients with primary APS when antiphospholipid antibodies became persistently negative.

The cycle is closing, indeed, as hitherto addressed, the genes which are the key for disease onset are also the novel ground for pharmacogenetic and pharmacogenomics, depicting new panoramas in the treatment of ADs [70]. We are entering in the era of personalized medicine in which the identification of individual pathogenic mechanism may allow tailored diagnostic pathways and treatments.

All these papers light the idea that unraveling the soul of autoimmune diseases is like playing chess, and with every discovery, we are adding new moves to the game. The pathogenesis, disease and treatment are the rook, bishop and knight pointing at checkmating ADs.

\section{References}

1. de Carvalho JF, Pereia RM, Shoenfeld Y. The mosaic of autoimmunity: the role of environmental factors. Front Biosci. 2009;1:501-9. 
2. Brickman CM, Shoenfeld Y. The mosaic of autoimmunity. Scand J Lab Invest Suppl. 2001;235:3-15.

3. Bogdanos DP, Smyk DS, Invernizzi P, Rigopoulou EI, Blank M, Pouria S, Shoenfeld Y. Infectome: a platform to trace infectious triggers of autoimmunity. Autoimmun Rev. 2012.

4. Perricone C, Agmon-Levin N, Ceccarelli F, Valesini G, Anaya JM, Shoenfeld Y. Genetics and autoantibodies. Immunol Res. 2013. doi:10.1007/s12026-013-8396-9.

5. Linn-Rasker SP, van Der Helm-van Mil AH, van Gaalen FA, et al. Smoking is a risk factor for anti-CCP antibodies only in rheumatoid arthritis patients who carry HLADRB1 shared epitope allels. Ann Rheum Dis. 2006;65:366-71.

6. Amaya-Amaya J, Sarmiento-Monroy JC, Mantilla RD, PinedaTamayo R, Rojas-Villarraga A, Anaya JM. Novel risk factors for cardiovascular disease in rheumatoid arthritis. Immunol Res. 2013. doi:10.1007/s12026-013-8398-7.

7. Bieber V, Cohen AD, Freud T, Agmon-Levin N, Gertel S, Amital H. Autoimmune smoke and fire-coexisting rheumatoid arthritis and chronic obstructive pulmonary disease: a cross-sectional analysis. Immunol Res. 2013. doi:10.1007/s12026-013-8395-x.

8. Bogdanos DP, Smyk DS, Invernizzi P, Rigopoulou EI, Blank M, Sakkas L, Pouria S, Shoenfeld Y. Tracing environmental markers of autoimmunity: introducing the infectome. Immunol Res. 2013. doi:10.1007/s12026-013-8399-6.

9. Kivity S, Agmon-Levin N, Blank M, Shoenfeld Y. Infections and autoimmunity. Trends Immunol. 2009;30:409-14.

10. Agmon-Levin N, Paz Z, Israeli E, Shoenfeld Y. Vaccines and autoimmunity. Nat Rev Rheumatol. 2009;5:648-52.

11. Shoenfeld Y. Infections, vaccines and autoimmunity. Lupus. 2009;18:1127-8.

12. Harnett W, Harnett MM. Molecular basis of worm-induced immunomodulation. Parasite Immunol. 2006;28(535-543): 12 .

13. Fischer S, Agmon-Levin N, Shapira Y, Porat Katz BS, Graell E, Cervera R, Stojanovich L, Gómez Puerta JA, Sanmartí R, Shoenfeld Y. Toxoplasma gondii: bystander or cofactor in rheumatoid arthritis. Immunol Res. 2013. doi:10.1007/s12026-013-8402-2.

14. Weinbrand-Goichberg J, Segal I, Ovadia A, Levine A, Dalal I Eosinophilic esophagitis: an immune-mediated esophageal disease. Immunol Res. 2013. doi:10.1007/s12026-013-8394-y.

15. Dolcino M, Zanoni G, Bason C, Tinazzi E, Boccola E, Valletta E, Contreas G, Lunardi C, Puccetti A. A subset of anti-rotavirus antibodies directed against the viral protein VP7 predicts the onset of celiac disease and induces typical features of the disease in the intestinal epithelial cell line T84. Immunol Res. 2013. doi: 10.1007/s12026-013-8420-0.

16. Lidar M, Shinar Y, Goldberg M, Ben-Zvi I, Langevitz P, Livneh A. E148Q MEFV mutation carriage and longevity in individuals of Ashkenazi origin. Immunol Res. 2013. doi:10.1007/s12026-0138414-y.

17. Shoenfeld Y, Agmon-Levin N. "ASIA"—autoimmune/inflammatory syndrome induced by adjuvants. J Autoimmun. 2011; 36:4-8.

18. Shoenfeld Y, Agmon-Levin N. Abstract of: 'ASIA'-autoimmune (auto-inflammatory) syndrome induced by adjuvant- a new syndrome to be defined. In: Abstracts of selected invited lectures. Lupus. 2011;20:340-7.

19. Shoenfeld Y. Autoimmune (autoinflammatory) syndrome induced by adjuvants provides a diagnostic framework for enigmatic conditions. The Rheumatologist. 2011;6:26-32.

20. Shoenfeld Y, Hughes GRV, Agmon-Levin N. The spectrum of ASIA: 'autoimmune (auto-inflammatory) syndrome induced by adjuvants'. Lupus. 2012;21:118-20.

21. Perricone C, Agmon-Levin N, Valesini G, Shoenfeld Y. Vaccination in patients with chronic or autoimmune rheumatic diseases: the ego, the id and the superego. Joint Bone Spine. 2012;79:1-3.
22. Levy Y, Rotman-Pikielny P, Ehrenfeld M, Shoenfeld Y. Silicone breast implantation-induced scleroderma: description of four patients and a critical review of the literature. Lupus. 2009;18:1226-32.

23. Israeli E. Gulf War Syndrome as a part of the autoimmune (autoinflammatory) syndrome induced by adjuvant (ASIA). Lupus. 2012;21:190.

24. Authier FJ, Sauvat S, Champey J, Drogou I, Coquet M, Gherardi RK. Chronic fatigue syndrome in patients with macrophagic myofasciitis. Arthritis Rheum. 2003;48:569-72.

25. Israeli $\mathrm{E}$. The new model of sick building syndrome: inclusion in ASIA or Shoenfeld's syndrome. Mod Rheumatol. 2012;22:802.

26. Israeli E, Agmon-Levin N, Blank M, Shoenfeld Y. Adjuvants and autoimmunity. Lupus. 2009;18:1217-25.

27. Agmon-Levin N, Zafrir Y, Paz Z, Shilton T, Zandman-Goddard G, Shoenfeld Y. Ten cases of systemic lupus erythematosus related to hepatitis B vaccine. Lupus. 2009;18:1192-7.

28. Shoenfeld Y, Aharon-Maor A, Sherer Y. Vaccination as an additional player in the mosaic of autoimmunity. Clin Exp Rheumatol. 2000;18:181-4.

29. Perricone C, Ceccarelli F, Valesini G. An overview on the genetic of rheumatoid arthritis: a never-ending story. Autoimmun Rev. 2011;10(10):599-608.

30. Perricone R, Perricone C, Shoenfeld Y. Autoimmunity: when the immune system becomes the self-ish giant. Autoimmun Rev. 2011;10(10):575-6.

31. Selmi C, Battezzati PM, Tishler M, Shoenfeld Y, Gershwin ME. Vaccines in the 21st century: the genetic response and the innocent bystander. Autoimmun Rev. 2005;4:79-81.

32. Cerpa-Cruz S, Paredes-Casillas P, Landeros Navarro E, BernardMedina AG, Martínez-Bonilla G, Gutiérrez-Ureña S. Adverse events following immunization with vaccines containing adjuvants. Immunol Res. 2013. doi:10.1007/s12026-013-8400-4.

33. Shaw CA, Tomljenovic L. Aluminum in the central nervous system (CNS): toxicity in humans and animals, vaccine adjuvants, and autoimmunity. Immunol Res. 2013. doi:10.1007/s12026-013-8403-1.

34. Harrington CR, Wischik CM, McArthur FK, Taylor GA, Edwardson JA, Candy JM. Alzheimer's-disease-like changes in tau protein processing: association with aluminium accumulation in brains of renal dialysis patients. Lancet. 1994;343:993-7.

35. Lopes MM, Caldas LQA. Young children with austim spectrum disorders: can aluminium bodyburden cause metabolism disruption? Toxicol Lett. 2011;205S:S60-179.

36. Banks WA, Kastin AJ. Aluminum-induced neurotoxicity: alterations in membrane function at the blood-brain barrier. Neurosci Biobehav Rev. 1989;13:47-53.

37. Prat AK, Biernacki K, Wosik K, Antel JP. Glial cell influence on the human blood-brain barrier. Glia. 2001;36:145-55.

38. Seubert A, Monaci E, Pizza M, O'Hagan DT, Wack A. The adjuvants aluminum hydroxide and MF59 induce monocyte and granulocyte chemoattractants and enhance monocyte differentiation toward dendritic cells. J Immunol. 2008;180:5402-12.

39. Luján L, Pérez M, Salazar E, Alvarez N, Gimeno M, Pinczowski P, Irusta S, Santamaría J, Insausti N, Cortés Y, Figueras L, Cuartielles I, Vila M, Fantova E, Chapullé JL. Autoimmune/autoinflammatory syndrome induced by adjuvants (ASIA syndrome) in commercial sheep. Immunol Res. 2013. doi:10.1007/s12026-013-8404-0.

40. Khan Z, Combadière C, Authier FJ, Itier V, Lux F, Exley C, MahroufYorgov M, Decrouy X, Moretto P, Tillement O, Gherardi RK, Cadusseau J. Slow CCL2-dependent translocation of biopersistent particles from muscle to brain. BMC Med. 2013;4(11):99.

41. Gherardi RK, Authier FJ. Macrophagic myofasciitis: characterization and pathophysiology. Lupus. 2012;21:184-9.

42. Carvalho JF, Barros SM, Branco JC. ASIA or Shoenfeld's syndrome: highlighting different perspectives for diffuse chronic pain. Acta Reumatol Port. 2011;36:10-2. 
43. Cohen Tervaert JW. Silicon exposure and vasculitis. In: Uversky VN, Kretsinger RH, Permyakov EA (eds). Encyclopedia of metalloproteins. Springer Science + Business Media, LLC. 2012. doi:10.1007/978-1-4614-1533-6.

44. Vera-Lastra O, Medina G, Cruz-Dominguez Mdel P, Ramirez P, Gayosso-Rivera JA, Anduaga-Dominguez H, Lievana-Torres C, Jara LJ. Human adjuvant disease induced by foreign substances: a new model of ASIA (Shoenfeld's syndrome). Lupus. 2012;21:128-35.

45. Shoaib BO, Patten BM. Human adjuvant disease: presentation as a 414 multiple slcerosis-like syndrome. South Med J. 1996;89: 179-88.

46. Nancy AL, Shoenfeld Y. Chronic fatigue syndrome with autoantibodies - the result of an augmented adjuvant effect of hepatitisB vaccine and silicone implant. Autoimmun Rev. 2008;8:52-5.

47. Cohen Tervaert JW, Kappel RM. Silicone implant incompatibility syndrome (SIIS): A frequent cause of ASIA (Shoenfeld's syndrome). Immunol Res. 2013. doi:10.1007/s12026-013-8401-3.

48. Tozzoli R, Sorrentino MC, Bizzaro N. Detecting multiple autoantibodies to diagnose autoimmune co-morbidity (multiple autoimmune syndromes and overlap syndromes): a challenge for the autoimmunologist. Immunol Res. 2013. doi:10.1007/s12026013-8418-7.

49. Damoiseaux J. Autoantibodies in the grocery shop: does quantity matter? Immunol Res. 2013. doi:10.1007/s12026-013-8419-6.

50. Ballanti E, Perricone C, di Muzio G, Kroegler B, Chimenti MS, Graceffa D, Perricone R. Role of the complement system in rheumatoid arthritis and psoriatic arthritis: relationship with antiTNF inhibitors. Autoimmun Rev. 2011;10(10):617-23.

51. Di Muzio G, Perricone C, Ballanti E, Kroegler B, Greco E, Novelli L, Conigliaro P, Cipriani P, Giacomelli R, Perricone R. Complement system and rheumatoid arthritis: relationships with autoantibodies, serological, clinical features, and anti-TNF treatment. Int J Immunopathol Pharmacol. 2011;24(2):357-66.

52. Szekanecz Z, Szabó Z, Zeher M, Soós L, Dankó K, Horváth I, Lakos G. Superior performance of the CCP3.1 test compared to $\mathrm{CCP} 2$ and $\mathrm{MCV}$ in the rheumatoid factor-negative RA population. Immunol Res. 2013. doi:10.1007/s12026-013-8425-8.

53. Zafrir Y, Gilburd B, Carrasco MG, Kivity S, Sánchez-Castañón M, López-Hoyos M, Mandel M, Szmyrka M, Shoenfeld Y, Agmon-Levin N. Evaluation of an automated chemiluminescent immunoassay kit for antinuclear antibodies in autoimmune diseases. Immunol Res. 2013. doi:10.1007/s12026-013-8416-9.

54. Pregnolato F, Chighizola CB, Encabo S, Shums Z, Norman GL, Tripodi A, Chantarangkul V, Bertero T, De Micheli V, Borghi MO, Meroni PL. Anti-phosphatidylserine/prothrombin antibodies: an additional diagnostic marker for APS? Immunol Res. 2013. doi:10.1007/s12026-013-8421-z.

55. Ballanti E, Perricone C, Greco E, Ballanti M, Di Muzio G, Chimenti MS, Perricone R. Complement and autoimmunity. Immunol Res. 2013. doi:10.1007/s12026-013-8422-y.

56. Chimenti MS, Perricone C, Graceffa D, Di Muzio G, Ballanti E, Guarino MD, Conigliaro P, Greco E, Kroegler B, Perricone R. Complement system in psoriatic arthritis: a useful marker in response prediction and monitoring of anti-TNF treatment. Clin Exp Rheumatol. 2012;30(1):23-30.

57. Kundzer AV, Volkova MV, Bogdanos DP, Rödiger S, Schierack P, Generalov I, Nevinsky GA, Roggenbuck D. Deoxyribonuclease activity of polyclonal IgGs: a putative serological marker in patients with spondyloarthritides. Immunol Res. 2013. doi: 10.1007/s12026-013-8424-9.

58. Padeh S, Farzam N, Chayen G, Gerstein M, Berkun Y. Pentraxin 3 is a marker of early joint inflammation in patients with juvenile idiopathic arthritis. Immunol Res. 2013. doi:10.1007/s12026-0138417-8.

59. Atzeni F, Turiel M, Hollan I, Meroni P, Sitia S, Tomasoni L, Sarzi-Puttini P. Usefulness of cardiovascular biomarkers and cardiac imaging in systemic rheumatic diseases. Autoimmun Rev. 2010;9(12):845-8.

60. Khattri S, Zandman-Goddard G. Statins and autoimmunity. Immunol Res. 2013. doi:10.1007/s12026-013-8409-8.

61. Katzav A, Bina H, Aronovich R, Chapman J. Treatment for experimental autoimmune neuritis with clodronate (Bonefos). Immunol Res. 2013. doi:10.1007/s12026-013-8406-y.

62. Le Dantec C, Alonso R, Fali T, Montero E, Devauchelle V, Saraux A, Pers JO, Renaudineau Y. Rationale for treating primary Sjögren's syndrome patients with an anti-CD6 monoclonal antibody (Itolizumab). Immunol Res. 2013. doi:10.1007/s12026-0138423-x.

63. Caso F, Iaccarino L, Bettio S, Ometto F, Costa L, Punzi L, Doria A. Refractory pemphigus foliaceus and Behçet's disease successfully treated with tocilizumab. Immunol Res. 2013. doi: 10.1007/s12026-013-8411-1.

64. Nalotto L, Iaccarino L, Zen M, Gatto M, Borella E, Domenighetti M, Punzi L, Doria A. Rituximab in refractory idiopathic inflammatory myopathies and antisynthetase syndrome: personal experience and review of the literature. Immunol Res. 2013. doi: 10.1007/s12026-013-8408-9.

65. Lora V, Bonaguri C, Gisondi P, Sandei F, Battistelli L, Russo A, Melegari A, Trenti T, Lippi G, Girolomoni G. Autoantibody induction and adipokine levels in patients with psoriasis treated with infliximab. Immunol Res. 2013. doi:10.1007/s12026-0138410-2.

66. Voswinkel J, Francois S, Gorin NC, Chapel A. Gastro-intestinal autoimmunity: preclinical experiences and successful therapy of fistulizing bowel diseases and gut Graft versus host disease by mesenchymal stromal cells. Immunol Res. 2013. doi:10.1007/ s12026-013-8397-8.

67. Proal AD, Albert PJ, Marshall TG, Blaney GP, Lindseth IA. Immunostimulation in the treatment for chronic fatigue syndrome/myalgic encephalomyelitis. Immunol Res. 2013. doi: 10.1007/s12026-013-8413-z.

68. Hershko AY, Scheiman-Elazari A, Aamar S, Naparstek Y. Extracorporeal immunoadsorption of antibodies against the VRT-101 laminin epitope in systemic lupus erythematosus: a feasibility evaluation study. Immunol Res. 2013. doi:10.1007/ s12026-013-8412-0.

69. Coloma Bazán E, Donate López C, Moreno Lozano P, Cervera R, Espinosa G. Discontinuation of anticoagulation or antiaggregation treatment may be safe in patients with primary antiphospholipid syndrome when antiphospholipid antibodies became persistently negative. Immunol Res. 2013. doi:10.1007/ s12026-013-8407-x.

70. Szekanecz Z, Meskó B, Poliska S, Váncsa A, Szamosi S, Végh E, Simkovics E, Laki J, Kurkó J, Besenyei T, Mikecz K, Glant TT, Nagy L. Pharmacogenetics and pharmacogenomics in rheumatology. Immunol Res. 2013. doi:10.1007/s12026-013-8405-z. 